\title{
Research on the Image Narrative Strategy Based on the Digital Vision - Thinking about the Creation of the Illustration Book "Yuxiang Yarn Factory"*
}

\author{
Ge Wan bao ${ }^{1 \dagger}$ \\ ${ }^{1}$ Hunan Normal University, Changsha, 41000, China
}

\begin{abstract}
With the transformation of media technology and social context in the new era, image provides auxiliary strategies for the diversified manifestations of culture in the process of communication. Traditional design drawings are increasingly unable to meet the new context requirements of modern architectural culture communication in terms of expression mode and acceptance by the public. As a new form of cultural dissemination or another way: digitization, imagery, narrative, and popular expression have begun to arouse discussion and thinking. With the help of digital technology as an expression of visual media, this paper tries to explore the potential of "image" as a medium to depict space, which brings about spatial situational experience and narrative expression. In addition, through montage and axonometric space operation, we explore the theory and practice in the narrative strategy of images, so as to build a bridge of communication between spatial experience of spatial consciousness and images. To be more precise, it emphasizes the connection between the image using digital technology as the medium and the spatial experience, and then uses the key of "imagine" to sort out the context of spatial awareness and emotional cognition.
\end{abstract}

\section{Introduction}

As visual text, image is not only a tool for expressing ideas, but also a traditional narrative medium. On the one hand, it narrates and describes the trajectory of human existence, and on the other hand, it plans and constructs people's cognition and expression. Therefore, images are not only a narrative method and medium, but also a form of visual design and expression.

As a new member of the image pedigree, digitized image is a visual presentation method spawned by the joint development of image technology and media technology. With the advent of the digital age, images have been given new life in the transmission of information and the diversity of visual media. ${ }^{[1]}$ As a model of image narrative, Illustration books are constantly changing in presentation methods, using digital means to expand from the dual perspectives of interaction and narration, which provides a direction for the protection and activation of Yuxiang Yarn Factory as a physical cultural symbol of Changsha city.

\section{Digital image narration expresses the cognition of spatial situation experience}

In the context of mainstream architectural graphics today, with the "professional" spatial thinking method, does it bury and constrain the image as a visual symbol in the process of communicating the potential value? How to express and convey the experience of architectural space through visual media? ${ }^{2]}$ This paper took the creation of the illustration book "Yuxiang Yarn Factory" as an entry point, and explored and studied the reproduction of historical blocks and industrial heritage buildings based on digital narrative images, and then used the urban architectural illustration book as an operating method to think about and explore the significance of architectural spaces (Illustration Book) expressed by digital images in the research and practice of contemporary architecture.

Due to the non-material characteristics of digital images, they must be presented in the form of digital display media, which is different from traditional architectural images and general computational images in terms of instrumentality. As a new creative method spawned by information technology, it not only shows people the infinite vitality of designed images in the new era, but also enhances the readability of audiences through narrative performance. Digital images mainly replace material materials with digital language, present the designer's design concepts and design effects in an allround way, and provide diversified strategies for digging and discovering the existence and clues of life in the space.

Digital images have expanded the form and visual language of human beings. The novel forms and styles provide the possibility for the transmission of new visual image information. It is realized and aroused by the public in the form of illustration books under digital media technology. The memory and cognition of architectural

\footnotetext{
* Corresponding author: 1056097102@qq.com
} 
heritage also provides a new perspective and thinking for the reproduction of history, space and culture. The way it promotes the general public's recognition and recognition of industrial architectural heritage is to arouse the audience's interest in the dissemination of culture. The memory of historical space is protected by culture, and development is promoted by culture. Protecting industrial heritage is to preserve a memory and a piece of history for the city ${ }^{1}$.

\section{Digital image reproduction of spatial experience-creative ideas for the illustration book of Yuxiang Yarn Factory.}

Robin Evans regards "picture" as a self-contained medium, and considers drawing as a tool and medium in architectural knowledge and architectural practice, thus discussing the relationship between different pictorial expressions and architecture. ${ }^{[3]}$ In the creation process of the illustration book "Yuxiang Yarn Factory", in order to create the meaning to be conveyed on the drawings, the research and exploration of operation techniques and expression tools are carried out, and the way to express the experience of space in a graphic manner is said to use the "illustration book" method to explore the understanding and perception of space, so as to seek the form and means of expression.

The illustration book summarizes the understanding of the story plot of Yuxiang Yarn Factory and the perception of historical memory, and summarizes it as an abstract symbol. Perhaps this abstract symbol is an extension of a certain meaning expressed in architectural drawings.

\subsection{Based on the creation concept of "Modernology"}

The purpose of the modernology is to return to the world of daily life and emphasize the living status of human beings as individuals with independent consciousness in society. This is consistent with the originality of the creation of the Illustration book "Yuxiang Yarn Factory" based on the industrial architectural heritage planning of the Yuxiang Yarn Factory. The creative idea of Illustration books relies on the theory of modernology to carry out images and videos of the life in the market of cotton mills, and more importantly, to consult and observe the precious cultural relics. ${ }^{[4]}$

In the creation of Illustration books, the form of the relationship between people and space, buildings, and spinning mills is embodied. It is the setting of the story plot of the characters in the image (speaking, thinking, emotion). The wonderful dialogue and delicate images attract the audience to experience every space in Yuxiang Yarn Factory, Illustration books use digital image narrative design to replace material materials with digital language, and present the designer's design concepts and

${ }^{1}$ Yuxiang Yarn Factory can be regarded as one of the "Old Eight Factory" among the industrial buildings in Changsha today, so it design effects in an all-round way. They derive the narrative structure form into the heuristic means of design thinking, and record the historical documents, the existing site and the oral survey records of the older generation of yarn factory workers, so as to realize the designer's combing and recording of design thinking, and the scene Narrative as the content expressed by images. (Figure 1)

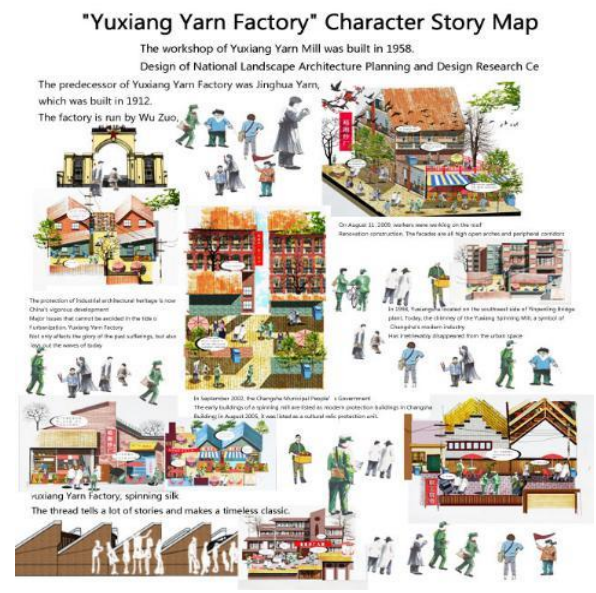

Figure 1: The story in the illustration book

\subsection{Scene construction and clue mining of "Yuxiang Yarn Factory"}

Taking the digital illustration book as the starting point, the design of its image, color, space and other styling elements in the form of expression needs to meet the basic requirements of narration. The narrative design tells the story through the design work (Illustration book). The "character" feature in the illustration book largely determines the development of the narrative. The visual morpheme design model also strengthens the "role" feature in the story to capture as much visual excitement as possible. Infect and persuade consumers by telling stories, making them an appreciator and user of the design work (Illustration book). ${ }^{[5]}$ The technical advantages of digitalization are better at shaping these visual morphemes, so it can give the characters and expressions rich in morphemes to make the narrative more substantial and fuller, so that the passive and silent "images" can arouse the emotional cognition of the audience, giving vitality to the originally cold industrial heritage buildings and become precious emotional sustenance.

Through the reference and investigation of data and the reproduction and drawing of real-life images, the daily life of the residents of the spinning mill is used as the theme in the setting of the storyline, and the clues of "people", "events" and "places" are combined with the film of "A big potato", the chief engineer Wang Xi developed a narrative space for the prototype of the character event, trying to use the picture book's more imagination and extension of the industrial architectural heritage to achieve a daily life full of Hosoi.

is of great significance to the study of Changsha's historical industrial heritage buildings. 
The digital image narrative strategy is mainly reproduced from four aspects:

1) Emotional expression-memory reproduction: through the historical deeds of "The Past and Present of Yuxiang Yarn Factory" written by Mr. Zhang Xiangtao and the oral data of the bridge section and the old employees of the spinning mill in the movie "A big potato".

2) Space Reorganization-Space Shaping: On-site modeling, measurement, and drawing of the actual buildings of the Yuxiang Yarn Factory, and digital modeling and reshaping using the reference of the leftover building groups and old photos. (Figure 2)

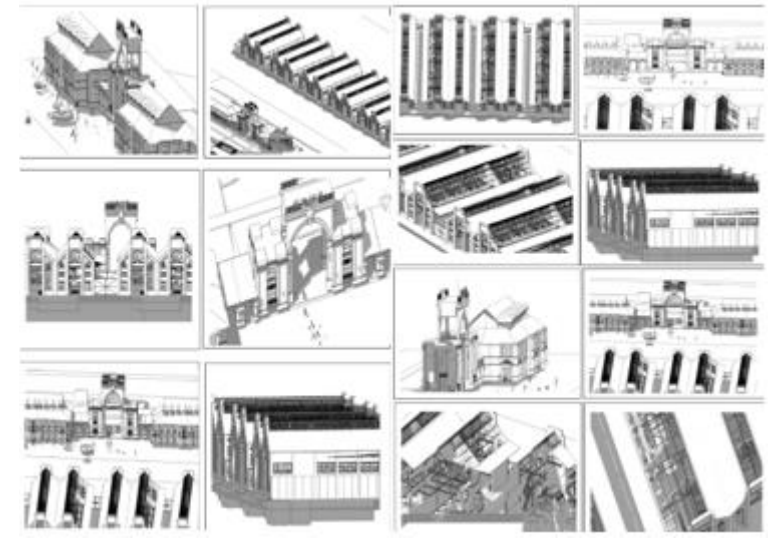

Figure 2: Model drawing of Yuxiang cotton mill

3) Narrative development-event presentation: Combine the protagonist, realistic story and architectural space in the movie bridge, and use the form of picture book to narrat

4) Historical return-panoramic narrative: through event interpretation, story reproduction activates the urban space and realizes the protection and rejuvenation of industrial architectural heritage. The author sorts out and evolves the relational reasoning diagram. ${ }^{2}$ (Figure 3 )

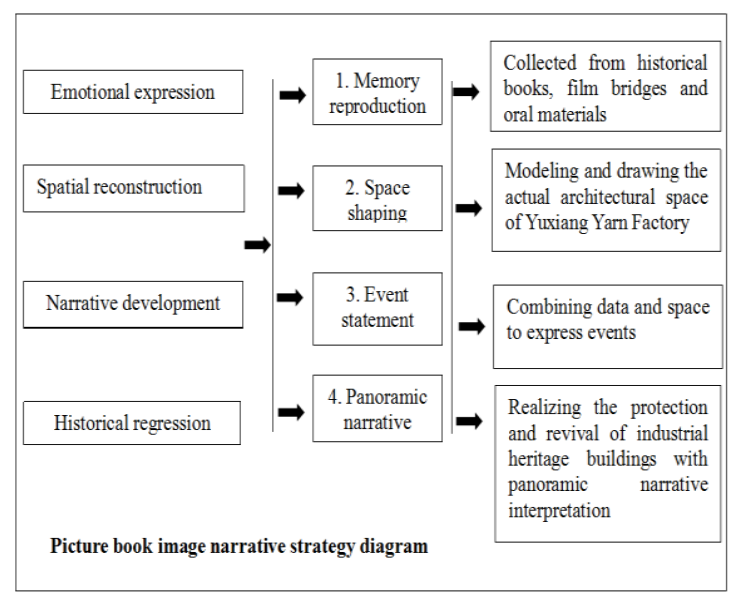

Figure 3: The narrative relationship strategy diagram of the illustration book "Yuxiang Yarn Factory"

\section{The diversified exploration of "graphic drawing" experience and narrative performance}

In the process of expressing and constructing digital image narrative, it is the experience of the painter in the spatial emotion that composes meaningful events to spread. In the narrative festival, life allusions, historical facts, and story emotions are all elements of its expression. In the creation process of the picture book "Yuxiang Yarn Factory", to better reflect these elements, the expression of digital image narrative was used to stimulate re-understanding of spatial situation. The color, shape, space and other symbols in the graphic expression are likened to a narrative energy store. At this time, the expression of spatial situation experience is carried out through the participation of these narrative symbols and the reader in the process of reading the picture.

\subsection{Essence of narration-The temporalization of space}

The essence of narration lies in the spatialization of time. How to give timeliness to images that are still or stagnant? In the process of image narration, it implies or reflects the movement of events, which is re-incorporated into the flow of time, making the image so unified. This kind of spatialized state becomes time slice to achieve the purpose of narrative movement. ${ }^{[6]}$

The reconstruction of time and space is to restore the context of the context through the image as a "slice" of time, to reconstruct the time flow of the image, which is the temporality in the Illustration book. The spatializationbased image reflects the dynamics of time, which makes the inanimate image produce a chain of causality in time. While constructing the concept of time for the image, it restores or reconstructs a narrative context. At this time, the image has begun to possess basic narrative ability.

How to achieve emotional resonance among painters, images and readers is also the value of studying Illustration books. The premise of creating a space experience that can be easily accepted and recognized by the public is to tap people's sensory experience of space and the emotional experience of memory. Therefore, when drawing digital images in a Illustration book, the painter subjectively incorporates his own emotional expression of life experience and space experience into the digital narrative image, which can make the dialogue between the painter and the reader more relaxed and effective, thereby when the audience accepting drawing information, making they got rid of the barriers caused by the so-called "specialization" expression.

\subsection{Manipulation method-Montage and axonometric space}

Montage $^{3}$, originally a term in architecture, refers to the

\footnotetext{
${ }^{3}$ Regarding the definition of montage, it is different. According to the "Dictionary of Cinematic Art" published by China Film Publishing House, the consensus among montage critics is that

\footnotetext{
2 "Modernology" is a modern school in Japan that investigates contemporary lifestyles and customs from various perspectives, with a special focus on Tokyo and the lives of local people.
} 
field of art, which means the subjective editing and collage of time and space. "Montage" as a creative method is often used in various fields, such as directors in film shooting, architects in the process of architectural design, etc., in order to make it rich in layers of meaning, the usual operation method is to use the lens or images and pictures to arrange in a purposeful and orderly manner, thereby deliberately creating a joint relationship. ${ }^{[7]}$

The montage technique not only enhances the tortuosity, interest, and richness of the space experience, but also creates suspense on the space plot, full of rhythm and rhythm changes. In the creation of picture books, to give a new context to the originally complex space-time relationship, in a limited space (Illustration books), the collage, combination, and overlap of events are used to express events of different time-space concepts into the same picture. It uses metaphor to construct images to express the main idea, and at the same time it forms a combination of montage-style small stories. ${ }^{[8]}$

Axonometric space is the use of software to produce axonometric scenes when digital image computer software (CAD) is used to draw drawings. Axonometric drawings squash the space, (telephoto) and flatten objects. This is similar to digital models. There is a certain difference in imaging. Axonometry gathers the non-space in the same time-space relationship to reproduce the complex picture of Yuxiang Yarn Factory. As a form of visual expression, this is irreplaceable for realistic photography. First, the lens cannot parallelize perspective. Even if an advanced shift camera can be used to correct vertical or horizontal distortion, it cannot do anything about the depth direction. ${ }^{[9]}$ Therefore, the irreplaceable visual experience produced by axonometric drawing enables it to surpass the function of engineering drawing and is a unique form of expressive art. Finally, it put the produced drawings into photoshop for coloring.

Before drawing, the author measured and framing the remaining gates and office buildings of Yuxiang Yarn Factory, and collected data on the parts that have disappeared and destroyed (factories, staff dormitories), and used digital software to restore the drawings, so as to help the space creation, step analysis and thinking sorting out, and make it an attractive place. The image reproduction strategy is used to germinate the experience of the space, so as to assist the early conception of the plan and the later transmission of information. (Figure 4)
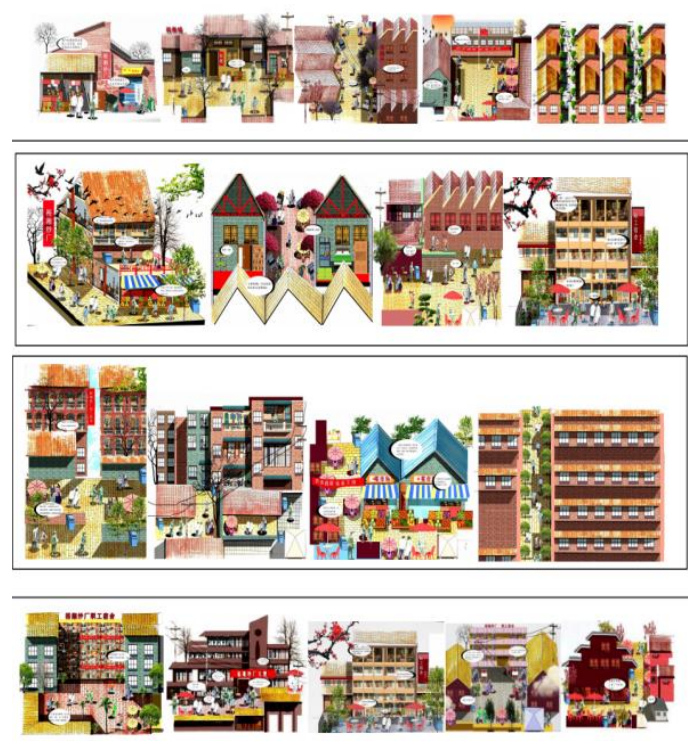

Figure 4: Partial drawings of Yuxiang Yarn Factory

\section{Prospects and Reflections on the Creation of Digital Illustration Book}

Urban illustration books have become a new strategy for the protection and revival of industrial heritage buildings, not only as a means of visual communication of images, but also as a visual language in our visual experience. However, to create expressions that are commonly accepted by the public and professionals, the use of multidisciplinary knowledge systems and operating techniques is one of the commonly used methods. As a new perspective to enrich human visual experience, the narrative representation of digital images needs to be deeply explored and reflected.

In the creation process of the illustration book "Yuxiang Yarn Factory", detailed research on the plots and regions was carried out. To better shape and describe the relationship between space and characters, the life situation of the employees of Yuxiang Yarn Factory was captured and imaged in the form of the exploration and experimentation of spatial reconstruction (illustration book reproduction). Through digital image expression, the historical style and life story situation of Yuxiang Yarn Factory can be reproduced, and easy-to-understand images are combined with familiar stories, urban industrial architecture, history, culture and art to form the image narrative of the re-recognition of the experience of the expression of spatial situation and thereby realizing the activation and regeneration of the industrial building heritage.

\section{ACKNOWLEDGMENTS}

From the topic selection of the thesis to the data collection and the establishment of the thesis framework, Professor

of thinking, that is: revealing the inner connection between things and phenomena, and understanding the essence of things through perceptual representation. the combination of shots is the source of the appeal of cinematic art, and the juxtaposition of two shots forms new characteristics and new meanings. Montage thinking conforms to the dialectics 
Lu Zheng of Hunan Normal University gave me a lot of suggestions and opinions. When my research direction was confused, Professor Lu Zheng patiently answered my questions and answered questions. It gave me a lot of valuable information and also gave me great motivation. In the site selection and measurement modeling and the creation of the picture book "Yuxiang Yarn Factory", I would like to thank Professor Wang Wei of Hunan University for his concern and guidance on the paper

\section{REFERENCES}

1. Shen Guandong; Research on Digital Image Narrative Design Mode [J]. Packaging Engineering, 59th Research Institute of China Ordnance Industry. 2016.02.08

2. (English) William W. Fweng-Spatial experience and construction before and after perspective [M]. Li Kairan. Nanjing: Southeast University Press, 2009.

3. (United States) by Jonathan Andrews. Architectural Conception - Contemporary Architectural Sketches, Perspective Drawings and Technical Drawings[M]. Yang Ting Luo Jia. Liu Dongyang School. Nanjing: China Construction Industry Press, 2010.

4. Wang Wei; Ou Xiongquan; Xu Haohao's Image Narrative and Reappearance in the Historic District- The Creation and Thinking of "Painting Changsha Taiping Street".-"Decoration"[J]-2018

5. (United States) Rudolf Arnheim. Art and Visual Perception. Teng Shouyao[M]. Translated by Zhu Jiangyuan·Sichuan People's Publishing House, 1998.

6. Long Diyong. Image Narrative-Temporalization of Space [J]. Jiangxi Social Sciences. 2007.09

7. Lu Shaoming Wang Bowei. Space Montage[J]. Beijing: World Architecture Magazine, 2005, (7).

8. [English]E $\cdot \mathrm{H} \cdot$ Gombrich. Art and Illusion: A Psychological Study of Image Reproduction[M]. Yang Chengkai, Li Benzheng, Fan Jingzhong Translated by Guangxi Fine Arts Publishing House, 2012

9. Wu Duan, Space Narrative Cognition: Motion Visual Experience Generated by Movie Real-time 3D Virtual Reality, Architect, 2008, 136(6): 43-49 